\title{
Horseshoe lung associated with a variant of the 'scimitar' syndrome
}

\author{
M. R. Dische, M. L. Teixeira, P. H. Winchester, and M. A. Engle \\ From the Departments of Pathology, Radiology, and Pediatrics, Cornell University Medical Center, New York, \\ New York I002I, U.S.A.
}

The rare anomaly of horseshoe lung with hypoplasia of the right lung in association with dextrorotation of the heart and anomalous drainage of the right pulmonary veins into the right atrium is presented. Similarity to the 'scimitar' syndrome and developmental considerations are discussed.

A combination of anomalies involving the pulmonary venous drainage has been designated the 'scimitar' syndrome because of its typical radiographic appearance of a large, curved venous trunk, usually parallel to the right heart border (Neill et al., I960; Kiely et al., 1967). In this condition the right pulmonary veins insert into the inferior vena cava, the right lung is hypoplastic, and the heart is displaced to the right. Usually there are also anomalous arterial connexions to the right lung (Frye et al., 1962).

Fusion of the two lungs into a 'horseshoe' arrangement, an extremely rare anomaly, has not previously been described in association with an anomalous pulmonary venous return. The only mention of a case of horseshoe lung appears in the textbook by Spencer (1968), who noted its association with cardiac dextroversion and an anomalous arterial supply to the hypoplastic right pulmonary component. He did not, however, describe any associated anomalies of the pulmonary venous return.

The object of the present report is to describe the various clinical and anatomical details and their implication in a case of horseshoe lung associated with anomalies closely resembling those observed in the 'scimitar' syndrome.

\section{Case report}

The infant, a Chinese boy, was born at term after his mother's second normal pregnancy. Birthweight was $3520 \mathrm{~g}$. He had an imperforate anus with a blind rectal pouch about $2 \mathrm{~cm}$ above the anal dimple and underwent double-barrel descending colostomy on the day after delivery. This was complicated by transient postoperative septicaemia (Esch. coli) which responded well to treatment. The additional anomaly of dextrocardia was noted on the initial physical examination and confirmed by $x$-ray examination (Fig. Ia). This showed dextrocardia with overcirculation of the lung, best seen on the left side, suggesting the possibility of anomalous venous drainage, perhaps 'scimitar' syndrome. In addition he had hemivertebra at L3. Cardiac examination disclosed an active heart with ventricular impact at the right sternal border. There was sinus tachycardia of 160 to 180 a minute. The first heart sound was normal; the second sound was narrowly split and of normal intensity. No thrill was present. Initially there was no heart murmur, but on the sixth day a $2 / 3$ systolic murmur along the left sternal border was first heard. It gradually became louder and radiated over the left hemithorax. Electrocardiograms were consistently abnormal, showing pronounced right axis deviation and abnormally deep $Q$ waves in leads I and II. Praecordial leads indicated that the right ventricle was normally dominant and that the heart was dextrorotated.

\section{Course}

The infant never regained his birthweight. He was discharged when 25 days old, but returned three days later with sudden onset of severe respiratory distress. He succumbed to irreversible cardiorespiratory arrest during readmission.

\section{Necropsy}

The body weighed $3420 \mathrm{~g}$. The double-barrel colostomy in the left upper quadrant was uninfected. A small fistulous tract was present in the perineum just anterior to the imperforate anal dimple. The abdominal and pelvic organs were normal except for the bulbous dilated blind end of the descending colon. The right umbilical artery was absent.

The heart was rotated to the right and its apex lay at the right anterior axillary line (Fig. Ib). The great arteries were normally disposed. The heart and lungs together 


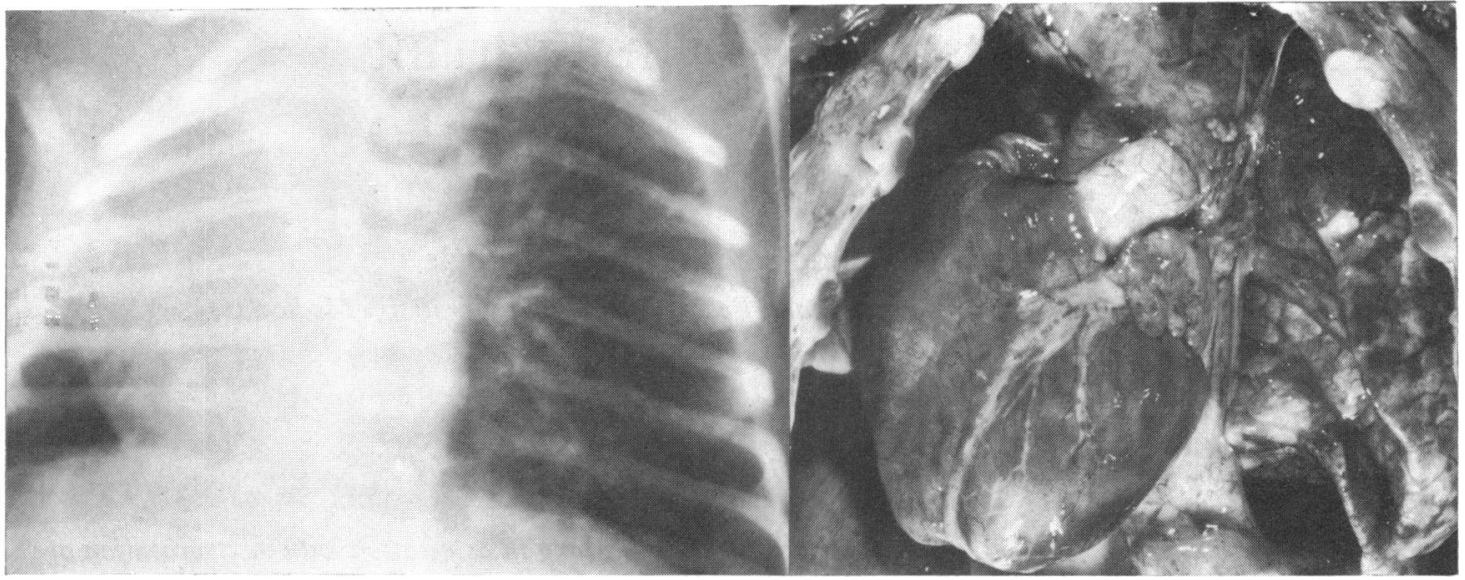

$a$

$b$

FIG. Ia) Chest X-ray: dextroversion of the heart and overcirculation of the left lung. b) Thoracic organs in situ: dextroversion of the heart.

weighed $105 \mathrm{~g}$; the heart alone about $30 \mathrm{~g}$. It was enlarged and the right ventricle was prominent. The coronary artery pattern was normal. The chambers of the heart were normally formed but the right ventricle was dilated. The veins from the right lung entered the right atrium between the ostia of the superior and inferior venae cavae. The fossa ovalis was a small funnel-shaped structure with a thick rim and a recessed septum primum which was patent anteriorly. The ventricular septum was intact. All valves were normal. The aortic cusps were finely fenestrated parallel to the line of closure.

The lungs were strikingly dissimilar in size. The small right lung measured $3 \mathrm{~cm}$ and the larger one $5 \mathrm{~cm}$ in their superior-inferior dimensions. The right lung was devoid of fissures; the left lung was bilobed. The posterior inferior portion of the right lung was continuous with a narrow bar or isthmus of normal appearing pulmonary tissue which crossed anterior to the aorta

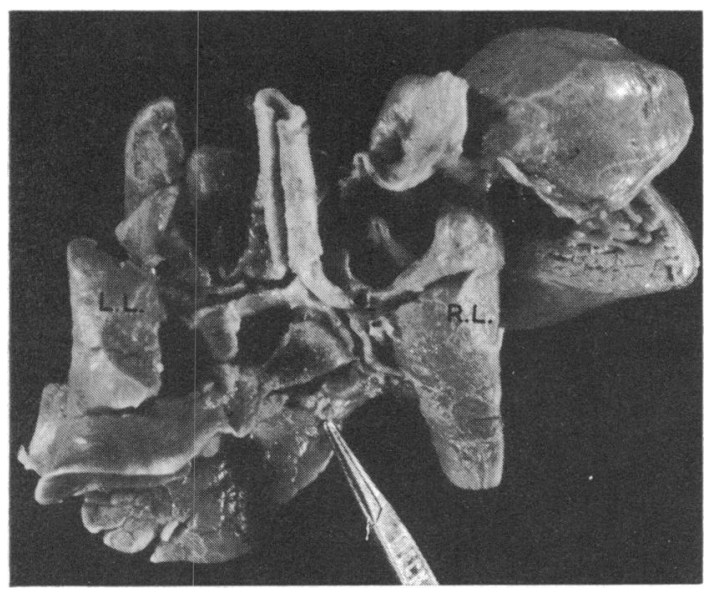

$a$

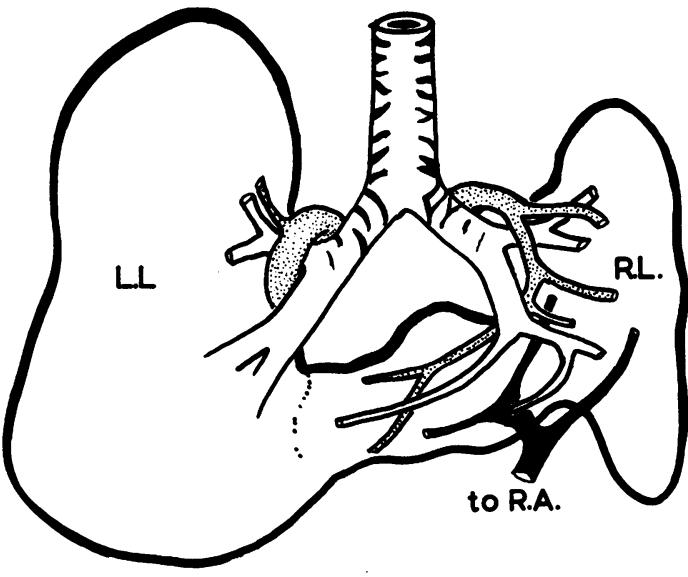

$b$

FIG. 2a) Posterior aspect of horseshoe lung: right bronchial branches supply isthmus; transected pulmonary artery crosses right upper bronchus. b) Schematic posterior view of horseshoe lung: pulmonary isthmus supplied by branches from the right bronchus and right pulmonary artery (stippled); bilateral hyparterial bronchi; anomalous right pulmonary venous drainage (solid black) to right atrium. 
and oesophagus at a level $1.5 \mathrm{~cm}$ below the carina, and was continuous with the posteromedial aspect of the left lower lobe. This isthmus measured $3 \mathrm{~cm}$ in length and from $I .5$ to $I .0 \mathrm{~cm}$ in thickness and width. The first bronchial branch on the left side arose at a distance of $I \cdot I \mathrm{~cm}$ from the carina and was hyparterial. Branches from the left bronchus supplied the left lung exclusively. The first right bronchial branch arose at a distance of $0.9 \mathrm{~cm}$ from the carina and was also hyparterial. The right main stem bronchus then bifurcated into two equal branches. One of these supplied the base of the right lung, the other the isthmus (Fig. $2 a$ and b). The main pulmonary artery divided unequally into a large left branch and a narrow but longer right branch. The ductus arteriosus was barely patent and its lumen practically sealed by prominent longitudinal wrinkles. The right pulmonary artery branch crossed over the first right bronchial branch, sending extensions to the right apex, to the right base, and to the isthmus. All veins from the hypoplastic right lung and the isthmus combined into a single trunk and drained in the right atrium as described. All veins from the left lung drained normally into the left atrium.

\section{Discussion}

The combination of malformations associated with the horseshoe lung in this case and in the case previously reported by Spencer, namely, hypoplasia of the right lung, dextroposition of the heart, anomalous pulmonary venous connexions, and anomalous arterial supply, closely resemble those in the 'scimitar' syndrome. However, in the present case, the only one that has been described in detail, all veins from the right lung drained directly into the right atrium.

According to Gasul, Arcilla, and Lev (1966), partial anomalous pulmonary venous drainage into the right atrium is accompanied in a large percentage of cases by defects in the atrial septum, a finding which may be explained by faulty development of the septum primum and subsequent incorporation of the venous connexion of the right lung into the right atrium. In the present case this explanation does not apply. The atrial septum was normal and the fossa ovalis was adequately covered by the septum primum. The fact that it was deeply recessed and anteriorly patent into the left atrium was undoubtedly secondary to increased flow to the right side of the heart, an interpretation that is supported by the large size of the right ventricle.

Normally, the anlage of the pulmonary veins is a dual one. The primitive pulmonary vein arises as an evagination from the cranial, atrial portion of the sinoatrial region of the heart, and the pulmonary venous connexions are elaborated by anastomoses with the rich splanchnic vascular plexus surrounding the primitive gut and long buds. The existence of two transitory evaginations in the caudal, sinus venosus portion of the sinoatrial region has been demonstrated in human embryos (Auër, 1948). The direct entry of the right pulmonary veins into the lateral border of an otherwise normally formed right atrial chamber may be interpreted as caused by abnormal persistence of the right caudal evagination of the sinus venosus. Anastomoses with the splanchnic venous plexus surrounding the abnormal posteriorly elongated right lung bud would then lead to drainage of all venous blood from the right lung to the right atrium.

Further similarity of this case to the 'scimitar' syndrome is to be found in the abnormal bronchial pattern. Halasz, Halloran, and Liebow (1956) noted that in carefully studied cases with this condition, anomalies in bronchial distribution were very frequent. In 66 per cent, bilateral left-sidedness was observed. This was also true in our case. The first bronchi arose almost equidistantly from the carina and both were clearly hyparterial. Their size was approximately equal. Similarly, symmetrical bronchial patterns have been frequently observed in combination with cardiovascular anomalies accompanied by polysplenia and frequently also by anomalous pulmonary venous drainage from the right lung to the right atrium (Landing and Wells, 1973; Van Mierop, Gessner, and Schiebler, 1972).

In most, but not all cases of the 'scimitar' syndrome, anomalous arteries from the aorta or its branches supply the entire right lung or a part. It is noteworthy that in the two cases of horseshoe lung available, one (Spencer, 1968) had an anomalous branch from the subclavian artery supplying the isthmic portion, while in the present case the right pulmonary arterial supply was normal. However, there was pronounced disparity in the size of the pulmonary arteries, the right one being long, narrow, and thin walled, while the left was shorter, wider, and thicker walled. We believe, therefore, that the overcirculation of the left lung and the relative undercirculation of the right lung, as seen radiographically, were truly present and not just obscured by the displaced large heart in the right hemithorax. It is noteworthy, however, that microscopical vascular changes seen in pulmonary hypertension were absent and that, in fact, the structure of the pulmonary arteries in both lungs was the same.

Malformed horseshoe lung appears to be a great rarity, though it may be occasionally overlooked at necropsy. In our own case, as well as in Spencer's, the fusion was situated inferiorly and consisted of an elongated isthmus which clearly belonged to the right lung, but was continuous with the parenchyma of the left lung without an intervening pleural layer. 
The structure of the isthmic parenchyma appeared normal. In the 'scimitar' syndrome, bronchiectasis and other pathological features are not uncommon in the hypoplastic right lung. However, our patient, the only one whose age was known, died within the first month of life while patients with the 'scimitar' syndrome who develop pulmonary lesions survive for longer periods.

It is not clear why fusion of the lungs should be of such extreme rarity, while fusion of the adrenals is rare (Warkany, 1971), and horseshoe or fused kidneys relatively common (Lenz, I964). It is possible that interposition of the large developing heart and of the aorta prevent such fusion, an interpretation that is supported perhaps by the fact that fused kidneys usually do not ascend to their normal position but override the bifurcation of the aorta (Caffey, 1972).

\section{References}

Auër, J. (1948). The development of the human pulmonary vein and its major variations. Anatomical Record, 101, 58I.

Caffey, J. (1972). Pediatric $X$-ray Diagnosis, 6th ed., p. 771 . Year Book Medical Publishers, Chicago.

Frye, R. L., Marshall, H. W., Kincaid, O. W., and Burchell, H. B. (1962). Anomalous pulmonary venous drainage of the right lung into the inferior vena cava. British Heart fournal, 24, 696.
Gasul, B. M., Arcilla, R. A., and Lev, M. (1966). Heart Disease in Children, p. 490. J. B. Lippincott, Philadelphia.

Halasz, N. A., Halloran, K. H., and Liebow, A. A. (1956). Bronchial and arterial anomalies with drainage of the right lung into the inferior vena cava. Circulation, 14, 826.

Kiely, B., Filler, J., Stone, S., and Doyle, E. F. (1967). Syndrome of anomalous venous drainage of the right lung to the inferior vena cava; a review of 67 reported cases and three new cases in children. American fournal of Cardiology, 20, 102.

Landing, B. H., and Wells, T. R. (1973). Tracheobronchial anomalies in children. In Perspectives in Pediatric Pathology, Vol. I, p. I. Ed. by H. S. Rosenberg and R. P. Bolande. Year Book Medical Publishers, Chicago.

Lenz, W. (1964). Krankheiten des Urogenitalsystems. In Humangenetik, Vol. III, p. 253. Ed. by P. E. Becker. Georg Thieme, Stuttgart.

Neill, C. A., Ferencz, C., Sabiston, D. C., and Sheldon, H. (1960). The familial occurrence of hypoplastic right lung with systemic arterial supply and venous drainage, 'scimitar syndrome'. Bulletin of the fohns Hopkins Hospital, r07, $\mathrm{I}$.

Spencer, H. (1968). Pathology of the Lung, 2nd ed., p. 73. Pergamon Press, Oxford.

Van Mierop, L. H. S., Gessner, I. H., and Schiebler, G. L. (1972). Asplenia and polysplenia syndromes. Birth Defects: Original Article Series, Vol. 8, p. 36. Williams and Wilkins, Baltimore.

Warkany, J. (197I). Congenital Malformations, p. 43I. Year Book Medical Publishers, Chicago.

Requests for reprints to Dr. M. R. Dische, Department of Pathology, Cornell University Medical Center, 1300 York Avenue, New York, N.Y. 10021, U.S.A. 